\title{
Echocardiographic and pathoanatomical characteristics of isolated left ventricular non-compaction: a step towards classification as a distinct cardiomyopathy
}

\author{
R Jenni, E Oechslin, J Schneider, C Attenhofer Jost, P A Kaufmann
}

\begin{abstract}
Aim-To determine clear cut echocardiographic criteria for isolated ventricular non-compaction (IVNC), a cardiomyopathy as yet "unclassified" by the World Health Organization. The disease is not widely known and its diagnosis mostly missed.

Methods and results-In seven out of a series of 34 patients with IVNC the in vivo echocardiographic characteristics were validated against the anatomical examination of the heart removed after death in four and due to heart transplantation in three patients. Four morphological criteria diagnostic for IVNC were found. (1) Coexisting cardiac abnormalities were absent (by definition). (2) A two layer structure was seen, with a compacted thin epicardial band and a much thicker non-compacted endocardial layer of trabecular meshwork with deep endomyocardial spaces. A maximal end systolic ratio of non-compacted to compacted layers of $>2$ is diagnostic. (3) The predominant localisation of the pathology was to mid-lateral (seven of seven patients), apical (six), and mid-inferior (seven) areas. The pathological preparations confirmed the echocardiographic findings. Concomitant regional hypokinesia was not confined to the non-compacted segments. (4) There was colour Doppler evidence of deep perfused intertrabecular recesses.

Conclusions-Four clear cut echocardiographic diagnostic criteria were established. It is suggested that the WHO classification of cardiomyopathies be reconsidered to include IVNC as a distinct cardiomyopathy.
\end{abstract}

(Heart 2001;86:666-671)

Keywords: isolated ventricular non-compaction; morphological criteria; cardiomyopathy; echocardiography; pathology

As classification serves to bridge the gap between ignorance and knowledge the aim of the present study was to define clear cut echocardiographic diagnostic criteria for isolated ventricular non-compaction (IVNC) validated by necropsy. ${ }^{12}$ Although IVNC has been known for more than a decade, it is still an "unclassified" cardiomyopathy according to the World Health Organization classification of the cardiomyopathies..$^{2-5}$ As a consequence, the diagnosis of this disorder is mostly missed because of lack of knowledge, despite its important prognostic implications for patients. ${ }^{6}$ Thus, as is true for every disease, classification of IVNC (as a distinct cardiomyopathy) would facilitate its diagnosis and most probably contribute to unmasking a much higher incidence of this disorder.

Early diagnosis of IVNC and correct management of such patients are crucial as the clinical manifestation is characterised by important morbidity and mortality caused by early heart failure, life threatening ventricular arrhythmias, and systemic embolic events. ${ }^{7}$

While in normal development the myocardium condenses and the intertrabecular recesses are reduced to capillaries, deep intertrabecular recesses communicating with the ventricular endocardium may evolve in some patients because of an arrest of compaction of the loose interwoven meshwork of myocardial fibres during intrauterine life. ${ }^{89}$
A similar persistence of non-compacted myocardium is frequently reported in patients with congenital left or right ventricular outflow tract obstruction and is referred to as "spongy myocardium" or "persisting sinusoids" that communicate with the coronary arteries. ${ }^{8-15}$

By contrast, IVNC is a genetically heterogeneous congenital disorder characterised by a pattern of excessively prominent trabecular meshwork and deep intertrabecular recesses in the absence of other structural heart diseases. ${ }^{41617}$ Furthermore, while the persisting sinusoids are enlargements of the coronary vessels (comparable with haemangioma), the recesses in IVNC have no connection with the coronary circulation. ${ }^{11} 18$ In fact they are recesses covered by endocardial lining continuous with the ventricular cavity, predisposing to local thrombus formation. Thus, not only clinically but also morphologically IVNC seems to be a distinct entity of cardiomyopathy although diagnostic criteria have not yet been established.

The aim of the present study was to define clear cut echocardiographic diagnostic criteria for IVNC and to validate them with pathoanatomical heart preparations.

\section{Patients and methods}

STUDY SUBJECTS

Seven of 34 adult patients with IVNC seen between 1986 and 1999-the largest series reported in the literature-and diagnosed 


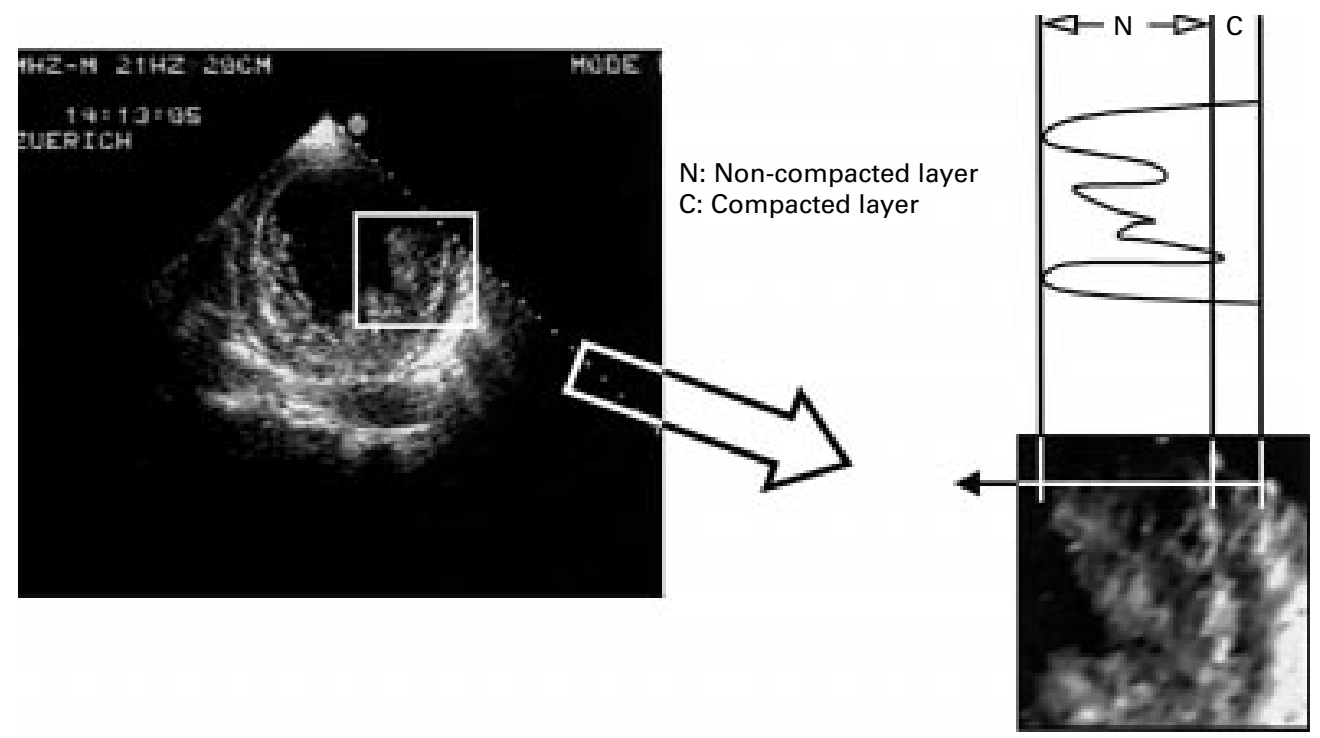

Figure 1 To quantify the extent of non-compaction at the site of maximal wall thickness the end systolic ratio of non-compacted to compacted thickness was determined. The two layers are best visualised at end systole as shown in this short axis view.

according to criteria previously published by our group were used in the present study. ${ }^{4} 18$ Hearts were accessible for anatomical study through heart transplantation (three patients) or postmortem examination (four patients), while no pathological anatomical examination was available for the remaining 27 patients. In addition, the echocardiographic findings of the seven IVNC patients were compared with the findings in nine patients with left ventricular hypertrophy ( $\mathrm{LVH}$ ) caused by hypertensive heart disease and with 10 patients who were evaluated and received transplants because of dilated cardiomyopathy (DCM) and of whom an anatomical study was available for comparison with the previous in vivo echocardiographic findings.

ECHOCARDIOGRAPHY

Two dimensional echocardiography was performed using an HP 77020AC (Hewlett Packard, Andover, Massachusetts, USA), HP Sonos 1500 (Hewlett Packard), VingMed 750 CFM (VingMed Sound, Horton, Norway), or Acuson Sequoia C256 (Acuson, Mountain View, California, USA). The following Doppler and two dimensional echocardiographic criteria diagnostic for IVNC were used as previously described by us and others:

- the absence of any coexisting cardiac anomalies;
- the characteristic appearance of numerous, excessively prominent trabeculations and deep intertrabecular recesses;

- intertrabecular spaces filled by direct blood flow from the ventricular cavity, as visualised on colour Doppler imaging. ${ }^{4} 1819$

Quantitative evaluation included two dimensional guided $\mathrm{M}$ mode standard measurements of the left ventricle according to the recommendations of the American Society of Echocardiography. ${ }^{20}$ Left ventricular biplane ejection fraction was calculated from an apical four chamber view and a right anterior oblique equivalent two dimensional echocardiographic view. ${ }^{2122}$ In the short axis view the site of end systolic myocardial wall thickness was assessed and in these areas the thickness of both the non-compacted and the compacted layer was determined (fig 1). For best visual differentiation of the two layers this measurement was performed during end systole. To quantify the extent of the non-compaction at the site of maximal thickness the end systolic ratio of non-compacted to compacted thickness was determined. As prominent trabeculations can also be found in LVH caused by DCM or hypertensive cardiomyopathy the differentiation from IVNC may be challenging. Therefore, the ratio of non-compacted to compacted layers in IVNC was compared with the ratio of trabeculated to untrabeculated dimensions in

Table 1 Patient characteristics

\begin{tabular}{lllllllll}
\hline Case & Sex & Age at diagnosis & $\begin{array}{l}\text { Age at death or } \\
\text { HTx }\end{array}$ & FS (\%) & EF (\%) & End point & $\begin{array}{l}\text { Mode of } \\
\text { death }\end{array}$ & $\begin{array}{l}\text { Heart weight } \\
(g)\end{array}$ \\
\hline 1 & M & 40 & 40 & 11 & 30 & HTx & - & $350^{\star}$ \\
2 & F & 52 & 55 & 10 & 20 & HTx & - & 560 \\
3 & M & 22 & 22 & 17 & 38 & Death & HF & 635 \\
4 & M & 19 & 29 & 33 & 27 & Death & SD & NA \\
5 & M & 61 & 61 & 11 & 23 & HTx & - & NA \\
6 & M & 54 & 56 & 18 & 30 & Death & HF & 850 \\
7 & F & 26 & 32 & 19 & 35 & Death & SD & NA
\end{tabular}

$\mathrm{EF}$, ejection fraction; F, female; FS, fractional shortening; HF, progressive heart failure; HTx, heart transplantation; M, male; NA, not available; SD, sudden death.

*Weight after fixation. 

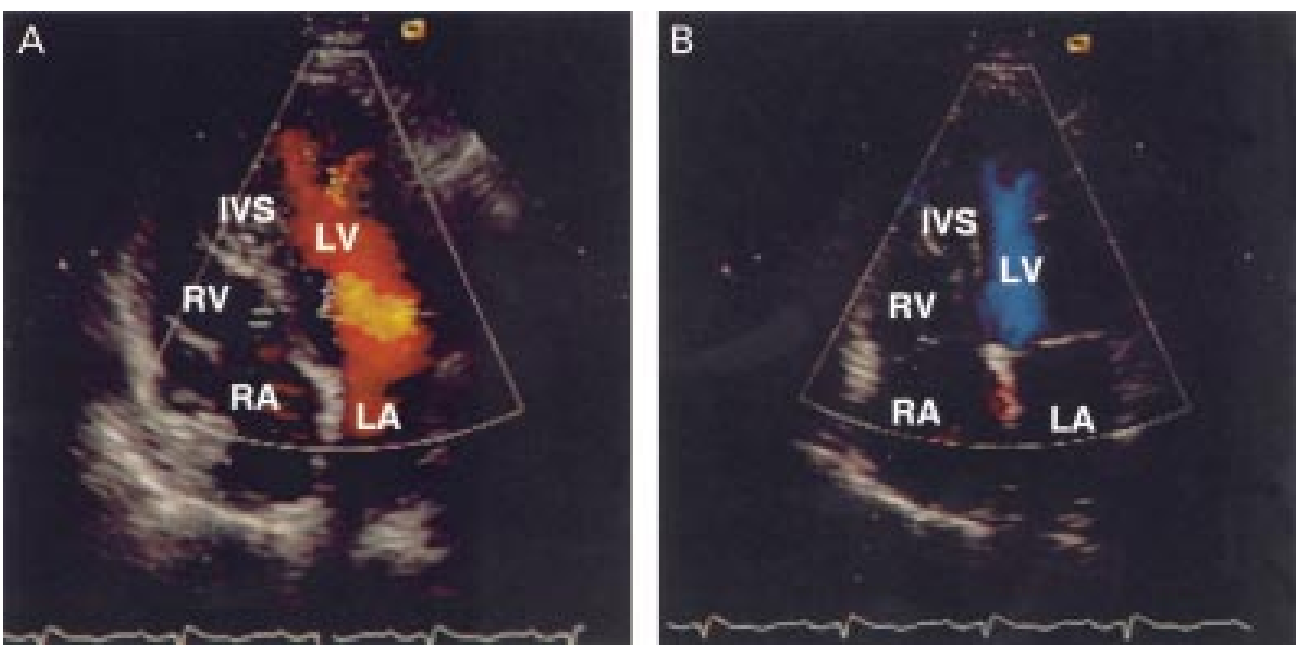

Figure 2 Colour Doppler study showed typical forward blood flow from the ventricular cavity into the deep spaces between the prominent trabeculation during diastole (in $A$ represented by a red signal) with a reversed flow back into the ventricle during systole (in B, blue signal).

10 patients with DCM and in nine patients with LVH caused by hypertension, despite the lack of a strictly speaking two layered structure in these two groups. However, there was no comparison with normal controls as it appeared futile owing to the lack of comparable characteristics.

The left ventricular wall was divided into nine segments: one apical, four midventricular, and four basal (septal, anterior, lateral, and inferior).

Because of the heavily trabeculated nature of the normal right ventricular apex we were reluctant to diagnose non-compaction in this ventricle. Thus, we cannot comment on right ventricular involvement as diagnosed by echocardiography.

PATHOLOGICAL AND HISTOLOGICAL ANALYSIS

After perfusion and fixation of the hearts with formalin, the macroscopic preparations were analysed. Special attention was given to obtaining a macroscopic pathological preparation cut corresponding to the four chamber or the short axis views used for the in vivo echocardiographic study to allow exact comparison and validation of the diagnostic imaging tool with the pathological anatomical findings. Histological sections of left ventricular myocardium were made with haematoxylin and eosin stains.

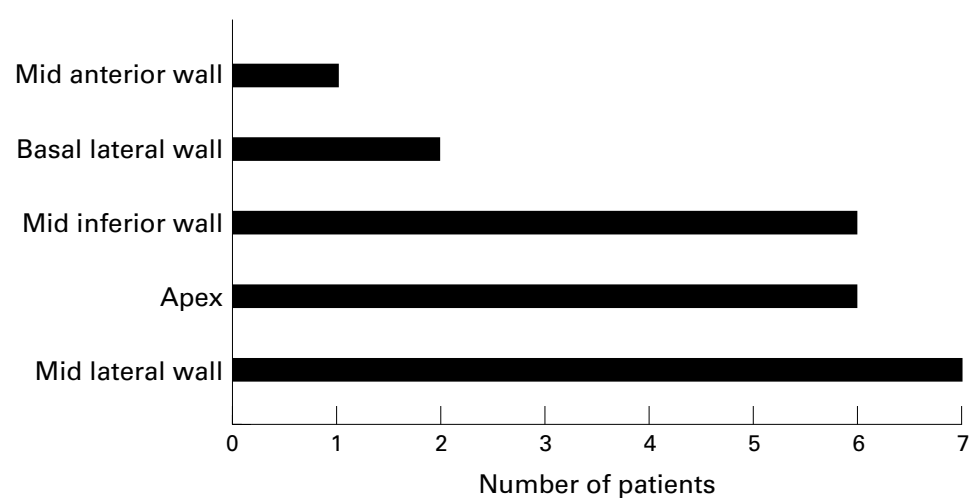

Figure 3 The structural alterations were predominantly localised to the left ventricular mid lateral wall and to the apex and the mid inferior wall.
STATISTICAL ANALYSIS

Echocardiographic measurements of wall thickness for the assessment of the ratio of non-compacted to compacted layers were made by two independent observers. The interobserver variation was analysed using Student's $t$ test. Results are given as mean (SD).

\section{Results}

PATIENT CHARACTERISTICS

The clinical characteristics are given in table 1 . In accordance with previous publications the predominant clinical features were heart failure, arrhythmias, and embolic events. Three patients received transplants because of end stage heart failure, and two died of sudden cardiac death and two of end stage heart failure.

ECHOCARDIOGRAPHY AND PATHOLOGY

Echocardiography

The affected segments had a two layer structure: a compact epicardial layer and an endocardial layer consisting of a prominent trabecular meshwork and deep intertrabecular spaces. This is best visualised in an end systolic short axis view (fig 1). The ratio of noncompacted to compacted myocardial layers at the site of maximal wall thickness averaged 3.5 (0.8) (range 2.3-5). The ratio of trabeculated to untrabeculated "layer" was $0.8(0.4)$ (range 0.4-2.0) in patients with DCM and $1.1(0.5)$ (range $0.4-2.0$ ) in those with LVH caused by hypertension (both $\mathrm{p}<0.001 v$ IVNC). The colour Doppler study showed typical forward and reversed direct blood flow from the ventricular cavity into the spaces between the prominent trabeculations throughout the cardiac cycle (fig 2).

In all seven patients the structural alterations were localised predominantly to the left ventricular mid-lateral wall, followed by the apex and the mid-inferior wall (fig 3). Decreased systolic thickening with wall motion abnormality (hypokinesis) was documented in all non-compacted segments of the left ventricular myocardium but also frequently found in the unaffected segments resulting in a 

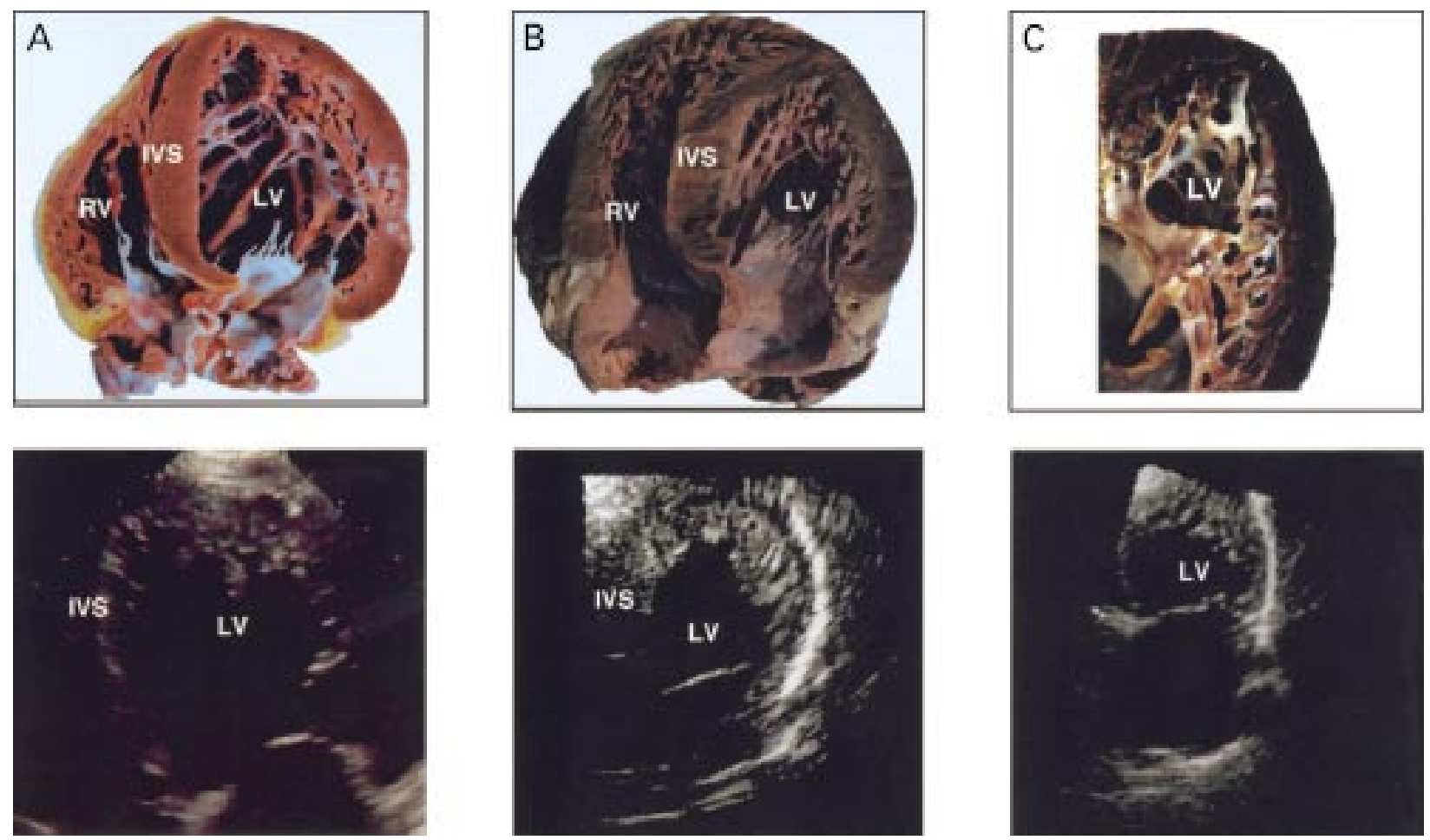

Figure 4 Apical four chamber view of three hearts. The anatomical findings (upper panel) are in agreement with the findings of the previously recorded echocardiographic view in the same patient (lower panel).

decreased global ejection fraction ranging from 20-38\% (mean 29 (6)).

\section{Pathology}

In IVNC all pathological anatomical examinations confirmed the localisation of the noncompacted myocardium corresponding to the echocardiographic findings. This is illustrated in an apical four chamber view (fig 4) showing the anatomical findings with their corresponding previously recorded echocardiographic recordings. None of the patients' hearts with an echocardiographic diagnosis of DCM showed any pathological anatomical feature of IVNC. Two patients showed involvement of the right ventricle with excessive trabeculation of the right ventricular apex.

\section{Histology}

Histological examination clearly confirmed that the recesses were covered by ventricular endocardium in continuity with the left ventricular cavity. Furthermore, ischaemic lesions were found in the thickened endocardium as well as in the prominent trabeculae surrounded by deep intertrabecular recesses (fig 5). Interstitial fibrosis was found to be severe in one, mild to moderate in four, and absent in one patient. Endocardial thickening in the left ventricular outflow tract was present in one patient. There was no fibre disarray in any of the cases. Signs of chronic inflammation were present in one patient. Abnormalities of intramyocardial blood vessels were found in two patients.

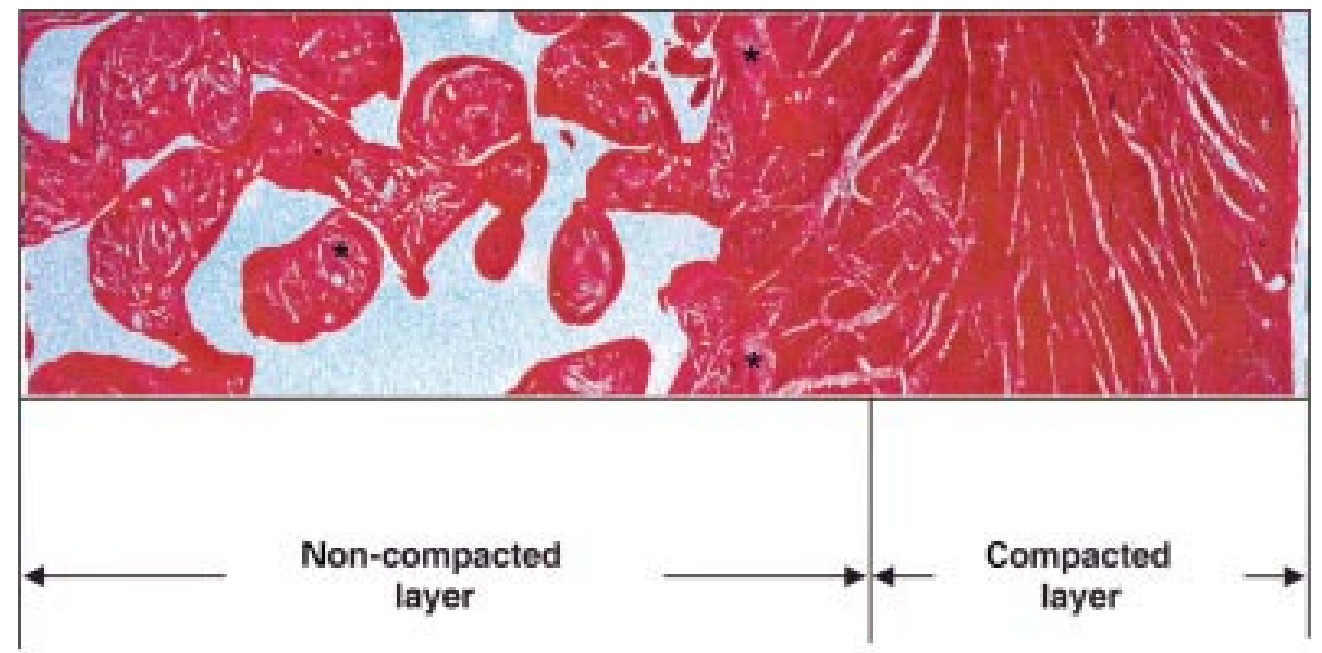

Figure 5 Histological preparation from the left ventricular apex of a patient with isolated ventricular non-compaction. Note the thin compacted normal outer layer of myocardium and the endocardial (non-compacted) layer. There is scar tissue within the trabeculations (asterisks) and in the subendocardial area but not in the epicardial zone. 


\section{Discussion}

According to the WHO definition cardiomyopathies are classified by the dominant pathophysiology or, if possible, by aetiological or pathogenetic factors. ${ }^{2}$ Although the cause of IVNC is not fully elucidated, the disease is thought to be a morphogenetic abnormality involving an arrest of compaction of the loose myocardial meshwork during fetal ontogenesis. This implies that IVNC should be present at birth in all patients, a notion supported by two previous reports. ${ }^{6}{ }^{19}$ Since IVNC has so far lacked a pathophysiological characterisation, this congenital anomaly has been unspecifically assigned to a heterogeneous group of "unclassified cardiomyopathies". This may contribute to the fact that the diagnosis of IVNC is mostly missed, as was true for almost $90 \%$ of the patients with IVNC in a series recently reported by Ichida and colleagues. ${ }^{6}$ Prominent left ventricular trabeculation can be found in healthy hearts as well as in hypertrophic cardiomyopathy and in $\mathrm{LVH}$ secondary to dilated, valvar, or hypertensive cardiomyopathy. Thus, the differentiation between variants and IVNC may often be challenging. As IVNC may have an important impact on morbidity and mortality, early and reliable diagnosis is crucial.

Therefore, we provide a characterisation of IVNC as a distinct entity of cardiomyopathy defining the following morphological criteria for diagnosis.

Coexisting cardiac anomalies that exclude non-compacted myocardium caused by excessively high pressure exposure of the ventricle during intrauterine development are absent. In particular, various forms of semilunar valve obstruction or left ventricular outflow tract obstruction have to be ruled out.

The thickened left ventricular wall consists of two zones of different structure. The compacted epicardial layer appears as a compact band of uniform tissue while the much thicker endocardial non-compacted layer consists of trabecular meshwork with deep endomyocardial spaces surrounded by exaggerated hypertrophy of the trabeculae. Although it must be emphasised that a strictly speaking two layered structure is found only in IVNC, and not in LVH, DCM, or any other condition, it may sometimes appear difficult to define where prominent trabeculation (such as in DCM) ends and non-compaction begins. However, an end systolic ratio of noncompacted to compacted layers of $>2$ is diagnostic for IVNC and allows unambiguous differentiation from hypertrophic cardiomyopathy and DCM or LVH.

The features of IVNC are found predominantly in the apical and the mid ventricular segments of the left ventricle, as confirmed in a larger series. ${ }^{18}$ Typically, these segments are hypokinetic contrarily to certain forms of apical cardiomyopathy that can otherwise mimic IVNC. However, hypokinesia may not be confined entirely to these segments but rather extend to morphologically unaffected segments, resulting in a decreased global ejection fraction. Although in the pathological examination involvement of the right ventricle was found in two cases, the heavily trabeculated nature of the normal right ventricle does not allow comment on right ventricular involvement as diagnosed by echocardiography.

Evidence of direct blood flow from the ventricular cavity into deep intertrabecular recesses by colour Doppler echocardiography is one of the hallmarks of the diagnosis of IVNC. This feature is clearly never observed in other forms of LVH.

Our study confirms that IVNC can be accurately diagnosed by echocardiography, as seen by the agreement with the necropsy findings. With regard to the exact ratio of noncompacted to compacted layers, however, comparison of the echocardiographic findings with the anatomical preparation is no more useful than it is-for obvious reasons-with regard to wall motion abnormalities. Although other modalities such as computer tomography, magnetic resonance imaging, and ultrafast computed tomography may also be helpful, no diagnostic criteria for these modalities have yet been proposed. ${ }^{63}$ We have established echocardiographic criteria for the diagnosis of IVNC in adults and validated the diagnostic accuracy against the pathological preparations, confirming and extending the criteria previously reported in children and adults. ${ }^{6} 71819$

Accurate diagnostic criteria are important for IVNC as its clinical morbidity includes heart failure caused by progressive ventricular dysfunction, arrhythmias, and systemic and pulmonary embolism. ${ }^{718} 19$ The latter may result from both impaired ejection fraction and from local thrombi within the deep intertrabecular recesses, as documented in one patient of our cohort. Such thrombi have to be searched for thoroughly as they are very often missed when associated with prominent trabeculations. ${ }^{24}$

\section{CONCLUSIONS}

For the first time, clear cut morphological criteria for the echocardiographic diagnosis of IVNC have been established, allowing accurate differentiation from other forms of LVH. There is evidence that this congenital anomaly is a distinct entity of cardiomyopathy with a characteristic morphological pattern. Therefore, we suggest that the WHO classification of cardiomyopathies be reconsidered to include IVNC as a distinct cardiomyopathy. This would improve not only the knowledge but also the awareness of this disorder and, thus, facilitate its diagnosis, as even a skilled echocardiographer's eye sees better while knowing what to look for.

Dr Philipp Kaufmann was supported by a grant from the Swiss National Science Foundation (SCORE B Grant-No. 3255002.98).

1 Goodwin JF. The frontiers of cardiomyopathy. Br Heart $\mathcal{F}$ 1982;48:672-3.

2 Richardson P, McKenna W, Bristow M, et al. Report of the 1995 World Health Organization/International Society and Federation of Cardiology task force on the definition and classification of cardiomyopathies. Circulation 1995;93: 841-2.

3 Engberding R, Bender F. Identification of a rare congenital anomaly of the myocardium by two-dimensional echocardiography: persistence of isolated myocardial sinusoids. Am f Cardiol 1984;53:1733-4. 
4 Jenni R, Goebel N, Tartini R, et al. Persisting myocardial sinusoids of both ventricles as an isolated anomaly: echocardiographic, angiographic, and pathologic anatomical findings. Cardiovasc Intervent Radiol 1986;72:675-80.

5 Allenby PA, Gould NS, Schwartz MF, et al. Dysplastic cardiac development presenting as cardiomyopathy. Arch Pathol Lab Med 1988;122:1255-8.

6 Ichida F, Hamamichi Y, Miyawaki T, et al. Clinical features of isolated noncompaction of the ventricular myocardium. f Am Coll Cardiol 1999;34:233-40.

7 Ritter M, Oechslin E, Sutsch G, et al. Isolated noncompaction of the myocardium in adults. Mayo Clin Proc 1997;72 $26-31$.

8 Grant RT. An unsusual anomaly of the coronary vessels in the malformed heart of a child. Heart 1926;13:273-83.

9 Bellet S, Gouley BA. Congenital heart disease with multiple cardiac anomalies: report of a case showing aortic atresia,
fibrous scar in myocardium and embryonal sinusoidal fibrous scar in myocardium and embryo
remains. Am $\mathcal{F}$ Med Sci 1932;183:458-65.

10 Dusek A, Osadal B, Duskova M. Postnatal persistence of spongy myocardium with embryonic blood supply. Arch Pathol 1975;99:312-7.

11 Angelini A, Melacini P, Barbero F, et al. Evolutionary persistence of spongy myocardium in humans. Circulation 1999;99:2475.

12 Davignon AL, DuShane JW, Kinacaid OW, et al. Pulmonary atresia with intact ventricular septum: report of two cases studied by selective angiocardiography and right heart catheterization. Am Heart 7 1963;62:690-7.

13 Elliot LP, Adams PJ, Edwards JE. Pulmonary atresia with intact ventricular septum. Br Heart $\mathcal{F}$ 1963;25:489-501.

14 Lauer RM, Fink RM, Petry EL, et al. Angiographic demonstration of intramyocardial sinusoids in pulmonary-valve atresia with intact ventricular septum and hypoplastic right ventricle. N Engl f Med 1964;271:68-72.
15 Hallman U, Mocellin R, Gössner W, et al. Ungewöhnliche Kontrastmittelverteilung im linksventrikulären Myokard Kontrastmittelverteilung im linksventrikulären Myokard bei Säuglingen mit hochgradiger valvulärer Aortenstenose und Endokardfibroelastose: pathologisch-morphologische Befunde. Z Kardiol 1983;72:675-80.

16 Gerull B, Sasse-Klaassen S, Oechslin E, et al. Isolated ventricular noncompaction of the myocardium is a genetically heterogeneous disorder. [abstract] Circulation 1999; 100:I-818.

17 Tsang JC, Chiu RC. The phantom of "myocardial sinusoids": a historical reappraisal. Ann Thorac Surg 1995; 60:1831-5.

18 Oechslin E, Attenhofer Jost CH, Rojas JR, et al. Long-term follow-up of 34 adults with isolated left ventricular noncompaction: a distinct cardiomyopathy. $7 \mathrm{Am}$ Coll Cardiol 2000;36:493-500.

19 Chin TK, Perloff JK, Williams RG, et al. Isolated noncompaction of left ventricular myocardium: a study of eight cases. Circulation 1990;82:507-13.

20 Devereux RB, Alonso DR, Lutas EM, et al. Echocardiographic assessment of left ventricular hypertrophy: comgraphic assessment of left ventricular hypertrophy: com-
parison to necropsy findings. Am 7 Cardiol 1986;57:450-8. parison to necropsy findings. Am 7 Cardiol 1986;57:450-8.
Jenni R, Vieli A, Hess OM, et al. Estimation of left ventricuJenni R, Vieli A, Hess OM, et al. Estimation of left ventricu-
lar volume from apical orthogonal 2-D echocardiograms. lar volume from apical ort

22 Nosir YF, Fioretti PM, Vletter WB, et al. Accurate measurement of left ventricular ejection fraction by threedimensional echocardiography: a comparison with radionuclide angiography. Circulation 1996;94:460-6.

23 Conces DJJ, Ryan T, Tarver RD. Noncompaction of ventricular myocardium: CT appearances. Am f Roentgenol 1991; 156:717-8.

24 Boyd MT, Seward JB, Tajik AJ, et al. Frequency and location of prominent left ventricular trabeculations at autopsy in 474 normal human hearts: implications for evaluation of mural thrombi by two-dimensional echocardiography. $\mathcal{F}$ Am Coll Cardiol 1987;9:323-6.

\section{IMAGES IN CARDIOLOGY}

\section{Echocardiographic demonstration of multiple coronary artery-left ventricular microfistulae}

A 68 year old woman was referred for evaluation of chest pain. The patient had a six month history of typical angina occurring with moderate exertion. She had a 10 year history of
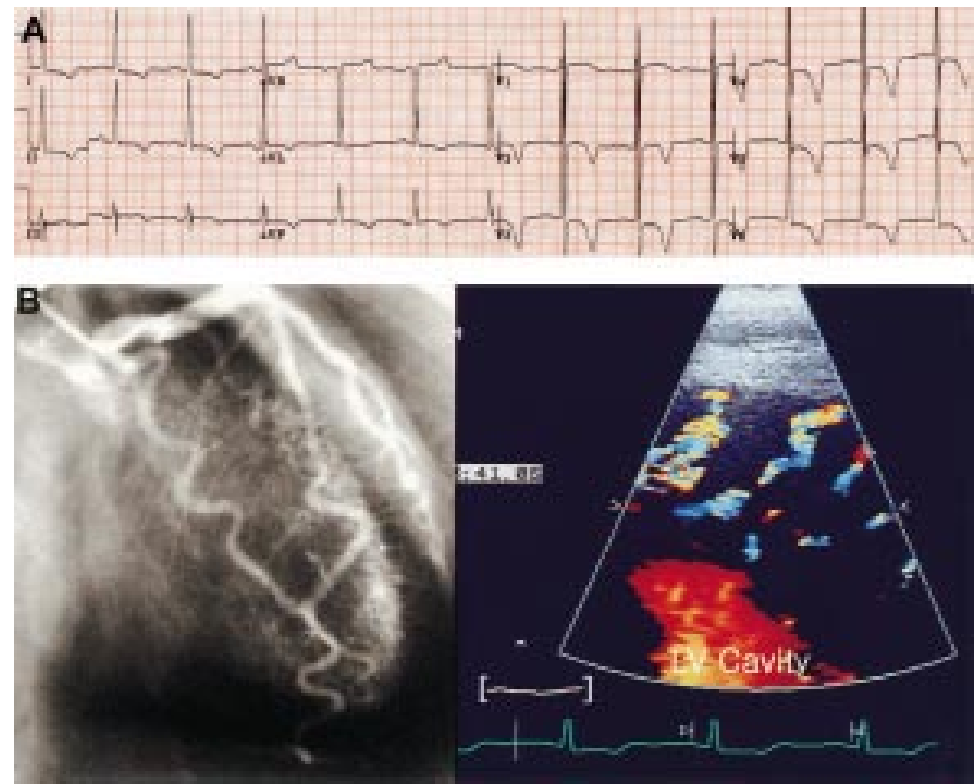

hypertension. Her blood pressure was 150/ $90 \mathrm{~mm} \mathrm{Hg}$. Precordial examination was normal with no murmur. The ECG recorded during chest pain showed symmetrical and deep $T$ wave inversion (A). Left ventriculography showed a normal sized left ventricle with an ejection fraction of $65 \%$. Left ventricular end diastolic pressure was $8 \mathrm{~mm} \mathrm{Hg}$. Coronary angiography revealed normal epicardial coronary arteries. After contrast injection the left ventricle was seen to fill faintly at end diastole with contrast from a diffuse plexus of fine vessels arising from the mid to distal portion of the left anterior descending coronary artery ( $\mathrm{B}, \mathrm{left})$. The coronary sinus was normal. Transthoracic colour Doppler echocardiogram just beneath the apical impulse window, using a high frequency transducer with a special preset coronary program with a low Nyquist limit, showed the presence of multiple linear colour flow signals perpendicular to the epicardial surface in the left ventricular apex, demonstrating penetrating intramyocardial coronary arteries arising from the left anterior descending coronary artery $(\mathrm{B}$, right).

HO-JOONG YOUN WOOK-SUNG CHUNG SOON-JO HONG younhj@cmc.cuk.ac.kr 